\title{
Chromosomal Translocation Engineering to Recapitulate Primary Events of Human Cancer
}

\author{
A. Forster, R. Pannell, L. Drynan, F. Cano, N. Chan, R. Codrington, A. Daser, \\ N. Lobato, M. Metzler, C.-H. Nam, S. Rodriguez, T. Tanaka, and T. RabbitTs \\ MRC Laboratory of Molecular Biology, Cambridge CB2 2QH, United Kingdom
}

\begin{abstract}
Mouse models of human cancers are important for understanding determinants of overt disease and for "preclinical" development of rational therapeutic strategies; for instance, based on macrodrugs. Chromosomal translocations underlie many human leukemias, sarcomas, and epithelial tumors. We have developed three technologies based on homologous recombination in mouse ES cells to mimic human chromosome translocations. The first, called the knockin method, allows creation of fusion genes like those typical of translocations of human leukemias and sarcomas. Two new conditional chromosomal translocation mimics have been developed. The first is a method for generating reciprocal chromosomal translocations de novo using Cre-loxP recombination (translocator mice). In some cases, there is incompatible gene orientation and the translocator model cannot be applied. We have developed a different model (invertor mice) for these situations. This method consists of introducing an inverted cDNA cassette into the intron of a target gene and bringing the cassette into the correct transcriptional orientation by Cre-loxP recombination. We describe experiments using the translocator model to generate MLL-mediated neoplasias and the invertor method to generate EWS-ERG-mediated cancer. These methods mimic the situation found in human chromosome translocations and provide the framework for design and study of human chromosomal translocations in mice.
\end{abstract}

The molecular pathology of cancer and possible new therapies have been a major concern of molecular biology since the first gene cloning experiments opened the possibility to clone, sequence, and study mutations in tumor cells. The very early observations of chromosomal changes in tumor cells (Boveri 1914) reflected genomic instability and raised the possibility of mutant proteins on the cancer cell surface to which antibodies might bind and elicit specific cell killing. There are a few examples of this, and most mutant proteins are firmly ensconced inside the cells and not available for antibody-mediated cell killing. Thus, although many mutations have been discovered, few specific therapies have been developed based on the molecular observations. Nevertheless, the cancer-specific mutations remain tantalizing targets for new cancer therapies.

Cancers have somatic mutations ranging from point mutations in genes like those of the $R A S$ family (for review, see Hanahan and Weinberg 2000), which create constitutively active signal transduction molecules (Fig. 1), to genes activated after chromosomal translocations, such as the CMYC gene in Burkitt's lymphoma, and to fusion genes created uniquely in cancer cells by chromosomal translocations, such as the $B C R-A B L$ gene fusion (for review, see Rabbitts 1994). As a range of genes involved in chromosomal translocations was identified, some common features began to emerge, especially in the acute cancers (hematopoietic and mesenchymal tumors) which invariably involve transcription factors whose normal role in cell fate decisions (Cleary 1991; Rabbitts 1991) is subverted (the master gene model; Rabbitts 1991). These transcription factors are often involved in protein-protein interactions. For instance, the T-cell leukemia genes LMO2 and TAL1/SCL are activated by distinct chromo- somal translocations, and their encoded proteins interact with each other to form a complex that binds to a bipartite site on DNA (Fig. 1). In addition, the recurrent finding of protein fusions resulting from chromosomal translocations (e.g., MLL or EWS fusions; Fig. 1) has again raised the specter of tumor-specific antigens for targeted therapies. However, the fact that many chromosomal translocation gene products are transcription factors, nuclear in location, and involved in protein-protein interactions moderates enthusiasm for them as therapeutic targets for small-molecule drugs.

New molecular therapeutic entities (macromolecular drugs), such as intracellular antibodies (intrabodies), siRNA molecules, or peptide aptamers (for review, see Rabbitts and Stocks 2003), could be therapeutic reagents based on the properties of, for instance, a chromosomal translocation gene product. However, there are many issues that need to be resolved before any possible use of such molecules (Rabbitts and Stocks 2003). Intrabodies can be effective in blocking protein-protein interactions and could form the basis of highly specific macromolecular drugs (Fig. 1). We have shown that single immunoglobulin variable region domains are most effective as intrabodies (IDAbs; Tanaka et al. 2003) and that the intra-chain disulfide bonds are dispensable for their function (Tanaka and Rabbitts 2003; Tanaka et al. 2003). Aside from the direct therapeutic use of intrabodies, macromolecular drugs of this type may serve as leads to identify small molecules (i.e., conventional drugs) that bind to the same place on the protein targets.

Animal models of spontaneous cancer will allow systematic evaluation of macromolecular drugs as well as leading to an understanding of the mechanism of tumorigenesis. It is not feasible to trial macromolecular drugs in 


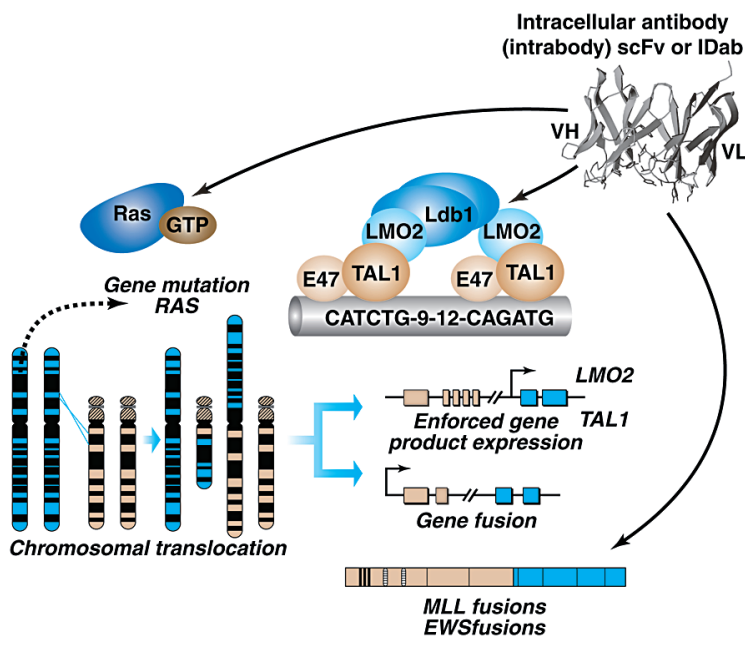

Figure 1. Mutations in cancer cells yield intracellular targets for therapy. Cancer cells have a number of mutations that contribute to the malignant phenotype. These may include point mutations in genes such as the $R A S$ family, encoding plasma membrane-associated GTP-binding molecules involved in signal transduction. Commonly, chromosomal translocations are present, especially in hematopoietic and mesenchymal tumors, that either result in oncogene activation or formation of a tumor-specific fusion gene (Rabbitts 1994). Examples of the former are LMO2 or TAL1/SCL activated in T-cell acute leukemia (forming multiprotein complexes involved in transcriptional regulation), and of the latter are $M L L$ or $E W S$-fusion genes found, respectively, in hematopoietic or mesenchymal (sarcoma) tumors. The proteins made from these mutant genes are attractive targets for therapeutics, for instance, utilizing novel entities such as intracellular antibody fragments (intrabodies) that can bind with high affinity to their targets in vivo. We have developed a method of Intracellular Antibody Capture (IAC) (Tse et al. 2002) to develop these reagents; and protocols appear at www2.mrc-lmb.cam.ac.uk/PNAC/Rabbitts_T/ group. ( $\mathrm{scFv}$ ) Single-chain Fv comprising a VH and a VL segment joined by a flexible linker region. (IDab) Single immunoglobulin domain $\mathrm{VH}$ or VL intrabody.

cancer patients due to the considerable challenge of developing methods of delivery to tumor sites and to limited numbers of suitable patients, together with concurrent use of conventional treatment. A preclinical strategy is outlined in Figure 2 that defines an initial therapeutic target from a chromosomal translocation, followed by development of a mouse cancer model based on that target. Independently developed anticancer reagents (e.g., intrabodies) can be tested for efficacy in the mouse model and parameters of delivery, toxicity, specificity, and effectiveness experimentally established, prior to any use in clinical settings.

The main options for making transgenic mouse models of human disease are indicated in Figure 3. The transgenic method is the conventional approach to make gain-offunction mice (Fig. 3A), and this can be an inducible system using antibiotic-responsive promoters. Alternatively, retroviral vectors can be used to deliver expression systems to target cells (Fig. 3B), followed by re-introduction into recipient mice. Our approach has been to use gene targeting by homologous recombination in mouse embryonic stem (ES) cells to make specific single genetic changes which form the basis of chromosomal transloca-

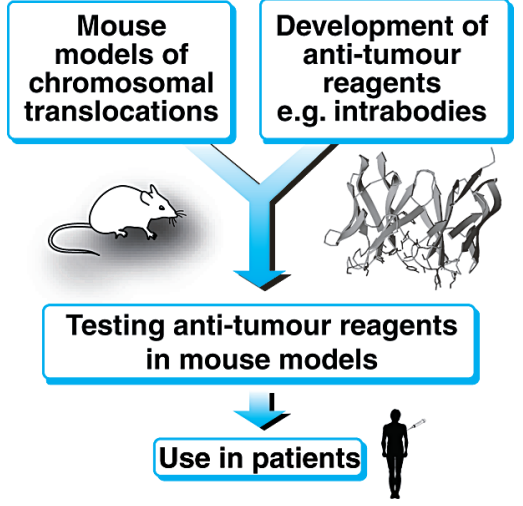

Figure 2. A strategy for preclinical testing of macrodrugs. Although mutations in cancer cells lead to expression of RNA and protein molecules that could be disrupted by macrodrugs, such as siRNA oligonucleotides or intracellular antibody fragments (intrabodies) (Rabbitts and Stocks 2003), there are major technical problems for their use in vivo. Model systems of tumor development, such as may be offered by mouse models of chromosomal translocations, are possible settings for development of novel methods to investigate use of molecular therapeutic entities (macrodrugs). Having first identified a target gene (e.g., via a chromosomal translocation), generating a mouse model that emulates the human cancer from which it was identified provides a preclinical test-bed for trialing new macrodrugs, prior to use in patients.

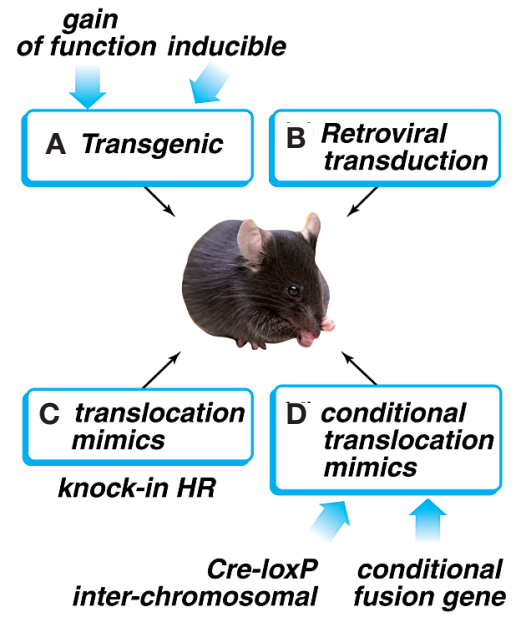

Figure 3. Options of developing mouse models of chromosomal translocations. Chromosomal translocation mimics can be made in various ways in mice. The most commonly used method to make mouse tumor models is transgenesis $(A)$. An alternative is retroviral transduction of target cells with a vector expressing the gene of interest $(B)$. Using homologous recombination (HR) in ES cells to generate targeted alleles offers an alternative approach to chromosomal translocation mimics. In an initial form, HR was used to knock-in a cDNA element into a target gene to achieve gene fusion $(C)$. Conditional forms of chromosomal translocation mimics can both recapitulate the lineage-specific synthesis of the translocation gene and allow conditional, cellspecific activation of chromosomal translocation genes $(D)$. We have used two methods: first, to create chromosomal translocations de novo in mice using Cre-loxP interchromosomal translocations (Collins et al. 2000; Forster et al. 2003), and second, to create chromosomal translocation mimics involving conditional formation of translocation fusion genes (Codrington et al. 2005; Forster et al. 2005). 
tion mimics or actual de novo chromosomal translocations (Fig. 3C,D, respectively). An advantage of using homologous recombination in ES cells is that the genetic changes made, when transmitted through the germ line of mice, are precisely defined by the format of the targeting event. If tumor formation occurs, it is because of the targeting event, together with any secondary mutations that may be necessary for overt disease to appear. Furthermore, targeting offers advantages over xenograft models, which are limited to immunodeficient hosts and generally involve growth of human cells on a mouse background.

We outline in this paper the development of methods for making mouse models of tumors arising from the equivalent of human chromosomal translocations. These models are translocation mimics involving knockin gene fusion, a conditional version or a direct recapitulation of interchromosomal translocations using recombination in mice.

\section{HOMOLOGOUS RECOMBINATION TO CREATE CHROMOSOMAL TRANSLOCATION MIMICS}

The most common consequence of reciprocal chromosomal translocations is gene fusion due to breaks within the introns of genes resulting in amalgamation into a chimeric gene, encoding a chimeric protein (for review, see Rabbitts 1994). We developed a simple approach to mimic the effect of gene fusion by knock-in of a cDNA element into the exon of a mouse target gene, equivalent to where a human translocation would occur (Corral et al. 1996; Dobson et al. 1999). We used the $M L L$ gene fusion with $A F 9$ as a model to establish the knockin approach to translocation mimics (Fig. 4). An $A F 9$ cDNA sequence was introduced into an $\mathrm{Mll}$ exon 10 gene targeting vector, together with a transcriptional termination polyadenyla-

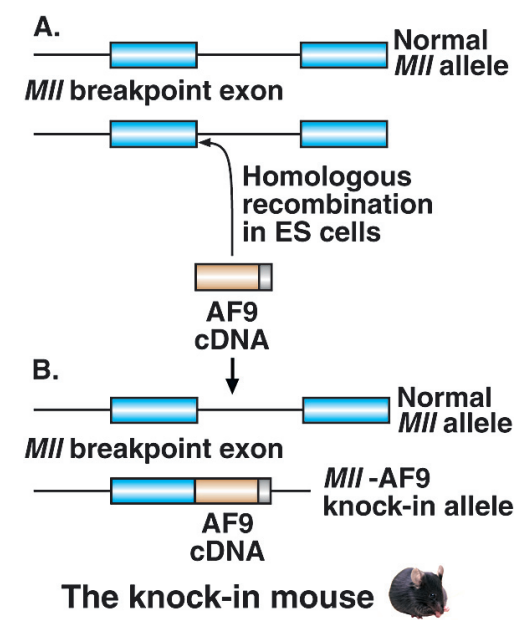

Figure 4. The Mll-AF9 knockin mouse model. Homologous recombination was used in ES cells to fuse a segment of $A F 9$ cDNA to an exon of the mouse $M l l$ gene $(A)$, each corresponding to the gene segments involved in the human chromosomal translocation $\mathrm{t}(9 ; 11)$ (Collins et al. 2000). The targeted ES cells are heterozygous for the Mll-AF9 gene fusion $(B)$ and mice made with these ES cells developed acute myeloid-lineage leukemia in 6-12 months. tion (pA) site and neomycin selectable marker, and the construct was used for homologous recombination in ES cells (Fig. 4A). ES cell lines were isolated with one normal $M l l$ allele and one targeted allele in which the $A F 9$ cDNA sequence was fused in-frame with Mll exon 10 (Fig. 4B). Mice were produced by injecting the targeted ES cells into blastocysts, and leukemias arose both in chimeric mice and in mice with germ-line transmission of the Mll-AF9 fusion gene (Corral et al. 1996; Dobson et al. 1999). This established the knockin procedure for making translocation mimics in mice.

The knockin fusion gene allele in mice is a germ-line mutation and should, in principle, be expressed wherever the promoter of the targeted gene is active. This is undesirable if the experimental protocol is designed for assessing the effects of the translocation mimic in specific tissues or within specific cells of a tissue. Knockin alleles can also have unexpected consequences, due to the gainof-function effects of expressing the fusion gene in embryos, resulting in embryonic lethality in some cases, such as observed for Mll-AF4 knockin (N. Lobata et al., unpubl.). These considerations make it desirable to create conditional forms of chromosomal translocation mimics; accordingly, we have developed two independent methods of doing this; namely, the translocator and the invertor mouse models.

\section{THE TRANSLOCATOR MOUSE MODEL OF DE NOVO CHROMOSOMAL TRANSLOCATIONS}

The Cre-loxP recombination system is very efficient and versatile to allow deletion, inversion, or translocation of DNA sequences (Hoess et al. 1982; Ramirez-Solis et al. 1995; Yu and Bradley 2001). The utility of this recombination system in generating chromosomal translocations in ES cells was shown for two independent selection systems (Smith et al. 1995; van Deursen et al. 1995), suggesting that interchromosomal translocations might be achievable between diverse loci in mice, offering an experimental system of de novo chromosomal translocations, conditional on Cre expression. We have tested the concept in mice using the equivalent of the human $M L L$ translocations, and our first studies showed that Mll-Af9 chromosomal translocations could be detected in peripheral tissues where Cre recombinase was expressed (Collins et al. 2000). We concluded that chromosomal translocations could be made de novo in mouse tissues and at sufficient frequency to be detected in the absence of any selection.

We further demonstrated that interchromosomal translocations could be made in mice resulting in development of hematopoietic tumors (Forster et al. 2003). LoxP sites were engineered into the appropriate introns of $M l l, A f 9$, and Enl genes, and mice were made from these targeted cells. Interbreeding of the mice produced lines with both $M l l$ and $A f 9$ loxP alleles (depicted as Mll-Afg translocator mice; Fig. 5A) or both Mll and Enl loxP alleles (depicted as Mll-Enl translocator mice; Fig. 5B). The effect of making de novo chromosomal transloca- 


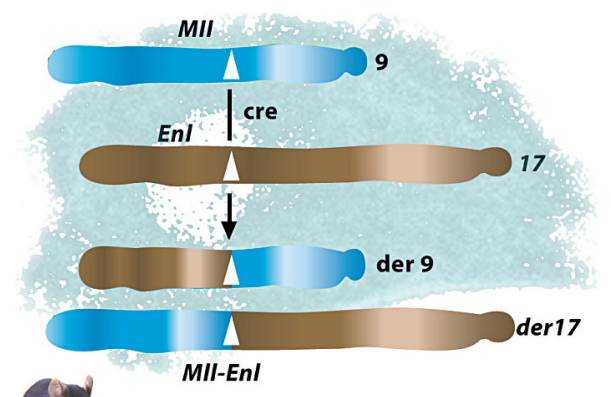

A. MII-Af9 translocator mouse

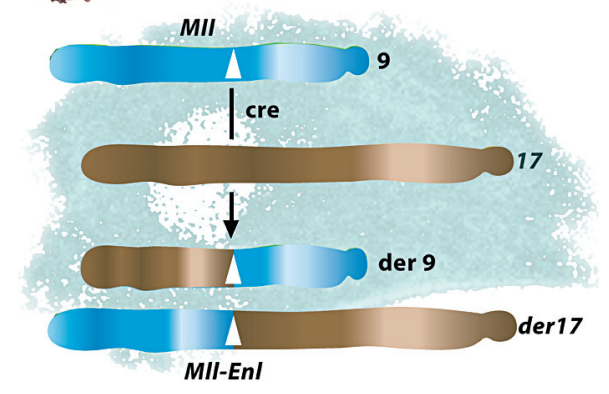

B. MII-EnI translocator mouse

Figure 5. Translocator model of chromosomal translocations. The Cre-loxP system was used to create de novo chromosomal translocations in mice. $\operatorname{Lox} P$ sites were recombined into appropriate introns of $M l l, A f 9(A)$ or $E n l(B)$ genes in ES cells. Mice carrying these alleles were made and interbred with each other and with mice expressing Cre recombinase (McCormack et al. 2003). Interchromosomal translocations occur de novo in cells expressing the Cre allele, equivalent to the human translocations $\mathrm{t}(9 ; 11)(A)$ and $\mathrm{t}(11 ; 19)(B)$, respectively. This translocator mouse model generates de novo reciprocal translocations and recapitulates the features of de novo human chromosomal translocations. LoxP sites are depicted by white triangles.

tions was studied via interbreeding mice expressing Cre in hematopoietic progenitors from a knockin of Cre in the Lmo2 gene (Fig. 6A). Both sets of compound genotype mice developed hematopoietic neoplasias (Fig. 6B shows the survival curves for the mice). Notably, the rate of tumor occurrence in $\mathrm{Mll}$; Enl; Lmo2-Cre mice was higher than in the corresponding Mll; Af9; Lmo2-Cre mice, and showed complete penetrance by 120 days. Considering that neoplasia was dependent on the presence of the Mll$E n l$ translocations, the short time to tumor appearance was remarkable and could mean that only the Mll-Enl fusion gene is necessary for overt disease in this model.

In both models, abundance of circulating myeloid lineage cells appeared, showing variable levels of maturity (Fig. 7A is a representative blood film from $\mathrm{Mll}$; Enl; Lmo2-Cre mice). The chromosomes from neoplasias of both $\mathrm{Mll}$; Enl and Mll-Af9 translocators include reciprocal chromosomal translocations demonstrated by fluorescence in situ hybridization (Fig. 7B shows painted metaphase chromosomes from a $\mathrm{Mll}$; Enl; Lmo2-Cre mouse tumor). Both of the translocator models shown in Figures 5 and 6 developed neoplasias of the myeloid lineage (Forster et al. 2003) (myeloproliferative disease-like [MPD] myeloid leukemia according to the proposed Bethesda classification [Kogan et al. 2002]).

These studies show that conditional formation of chromosomal translocations in hematopoietic progenitor cells, using an Lmo2-Cre knockin allele, can result in myeloid neoplasia (Fig. 7C) and that a similar type of disease occurs in the presence of the fusion gene made by the translocation of Mll-Enl or Mll-Af9. These models therefore emulate the human disease counterparts that arise from these fusions resulting from $\mathrm{t}(11 ; 19)$ and $\mathrm{t}(9 ; 11)$, respectively. The translocator approach therefore should allow the study of $M L L$ gene fusions in other specific cells and lineages in hematopoiesis by using the appropriate specific expression of Cre recombinase. In addition, the translocator model is a recapitulation of the primary events of human cancers by allowing the formation of reciprocal translocations in single cell precursors of the overt tumor. This should provide an ideal setting for testing drugs and new approaches, such as the intrabody technology mentioned briefly earlier (Fig. 1). As a first step, it will be valuable to ascertain whether the translocator mice with the $\mathrm{Mll}$ fusions respond to the chemotherapies currently used for treating the equivalent childhood acute leukemia.
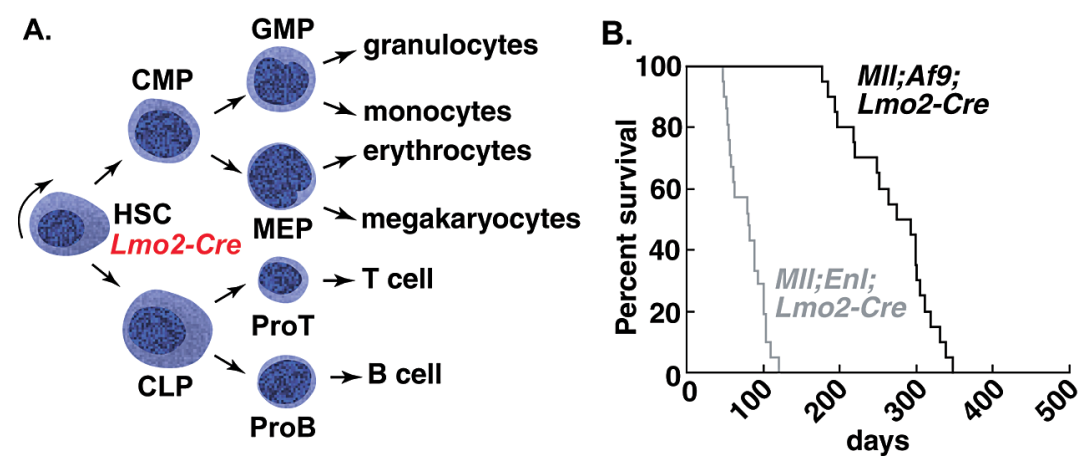

Figure 6. Leukemias occur in Mll-Af9 and Mll-Enl translocator mice. As described in Fig. 5, loxP sites were incorporated into $M l l$, Af 9 , and $E n l$ mouse genes facilitating the interchromosomal translocation mediated by Cre recombinase. In the experiments described here, Cre was produced from a knockin allele in the Lmo2 gene, driving Cre expression in hematopoietic progenitor cells (McCormack et al. 2003) (A shows a simplified pathway of hematopoiesis indicating pluripotent, self-renewing progenitor cells [HSC] giving rise to myeloid and lymphoid progenitors, respectively CMP and CLP, and in turn to mature, committed cells of myeloid and lymphoid lineage). Leukemias arise in both $M l l-E n l$ and $M l l-A f 9$ translocators ( $B$ shows Kaplan-Meyer survival curves for the two groups). 
Figure 7. Myeloid leukemia in Mll-Af9 and MllEnl translocator mice have reciprocal chromosomal translocations. Both Mll-Enl and Mll-Af9 translocator mice develop myeloid leukemias (MPD-like acute myeloid leukemia according to the Bethesda classification), characterized by typical pathology and circulating myeloid cells of varying maturity ( $A$ shows a blood smear from an Mll-Enl; Lmo2-Cre translocator). The tumor cells in these mice show normal ploidy and carry reciprocal chromosomal translocations ( $B$ shows a metaphase spread from an Mll-Enl; Lmo2-Cre tumor with $\mathrm{Mll}$ and $\mathrm{Enl}$ chromosomes painted green and red, respectively). Our findings show that the fusion of $M l l$ with either Enl or Af9 in hematopoietic progenitors results in tumors of the myeloid lineage $(C)$, typical of the types of tumors found in humans with these translocations. The translocator model recapitulates these primary events in human cancer.
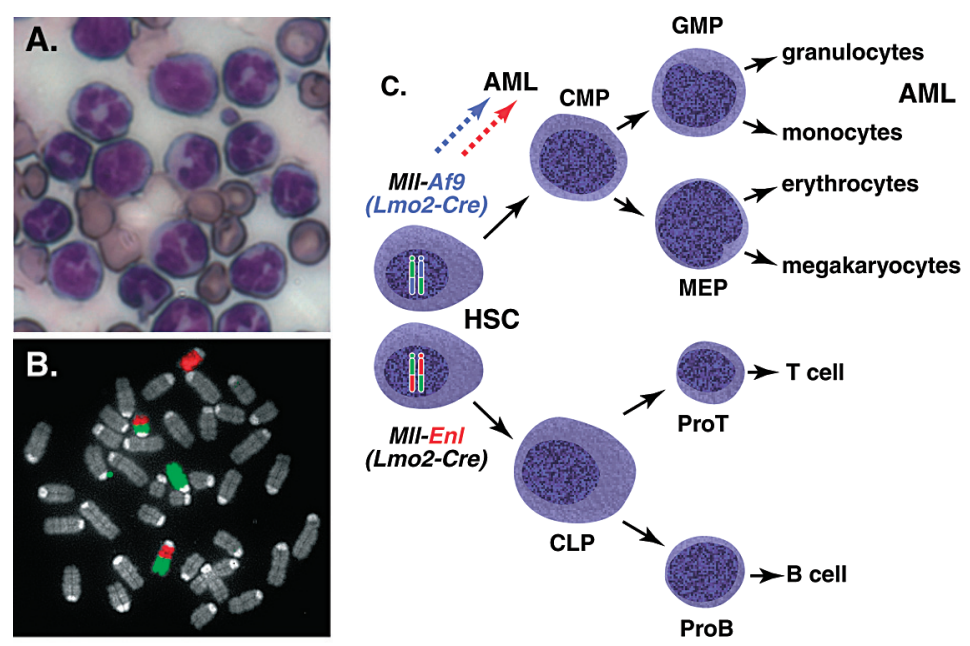

\section{ISSUES CONCERNING THE TRANSLOCATOR MODEL}

The translocator model relies on homologous recombination to insert loxP recombination sites into introns of interest to potentiate the formation of interchromosomal translocations in a desired cell type using specific $\mathrm{Cre}$-expressing alleles. In principle, this should be adaptable to mimic any human reciprocal chromosomal translocation. There are three main caveats to this proposition. First is that the spatial, nuclear organization of mouse chromosomes may preclude certain interactions that could support interchromosomal translocations. Second, the prevailing dogma is that genes should be accessible before a genomic rearrangement can take place, implying that only certain chromosomal regions could interact and be translocated.

A third potential problem is gene orientation. In the cases described here (i.e., $M l l, E n l$, and $A f 9$ ), the relative transcriptional orientation on each respective chromosome arm is the same as depicted in Figure 8A. This situation is compatible with the formation of two reciprocal chromosomes from a loxP-Cre-mediated translocation. However, if the two genes to be translocated are in the opposite transcriptional orientation (as depicted in Fig. 8B), loxP-Cre-mediated recombination would result in two abnormal reciprocal chromosomes, one dicentric and the other lacking a centromere. These chromosomes would be lost at mitosis and the cell would presumably not be viable. In these situations, the mouse translocator model would not be applicable and a different conditional translocation mimic is needed. For this purpose, we developed the invertor model (see below).

\section{THE INVERTOR CHROMOSOMAL TRANSLOCATION MIMIC}

To generate a fully conditional fusion gene model, we have made use of the ability of Cre recombinase to invert sequences flanked by appropriately oriented loxP sites, and developed the invertor model of chromosomal translocations (Codrington et al. 2005; Forster et al.
2005). In this approach, an inverted cassette, flanked by lox $P$ sites, is knocked into the intron of the gene to which a gene fusion is required (e.g., Ews as described below). The cassette comprises a short region of intron including an acceptor splice site, a cDNA segment for fusing with the target gene, a pA site for transcription termination, and the MC1-neo-pA fragment for selecting homologous recombinant ES cells. The invertor cassette is knocked in with the opposite transcription orientation to the target gene (Fig. 9A, inactive form). When mice are made from the invertor ES cells and crossed with Cre-expressing strains, the $\operatorname{lox} P$ sites flanking the invertor cassette are sites for recombinational inversion of the cassette, to make the functional form of the invertor allele (Fig. 9A, active form). In this configuration, transcription of the invertor allele proceeds from the target gene promoter to the pA site in the cassette, forming a pre-mRNA of ex-
A.

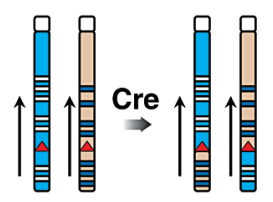

B.

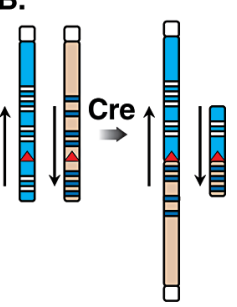

Figure 8. The translocator model is dependent on gene orientation. The translocator model can, in principle, recapitulate any human chromosomal translocation. However, mouse chromosomes are acrocentric (the centromeres are located at one end of each chromosome) and the orientation of the genes to be translocated is crucial to the success of the translocator strategy. In the situation depicted in A (such as the case of Mll-Enl and Mll-Af9), the gene orientation on each chromosome is identical, and therefore Cre-loxP-mediated interchromosomal translocation maintains the chromosomal organization of the genes on the derivation chromosomes. Conversely, if the genes are oriented in the opposite transcriptional direction $(B)$, Cre-lox $P$-mediated interchromosomal translocation results in two abnormal chromosomes (one dicentric and one lacking a centromere) unable to participate in mitosis. LoxP sites are depicted by red triangles and the centromeres by open boxes. 
A.

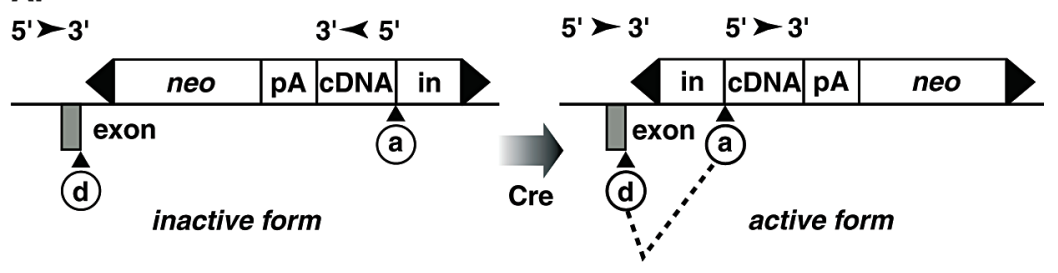

B.
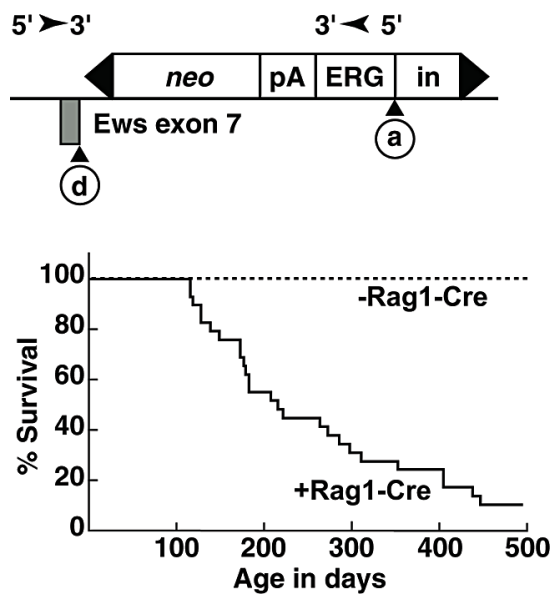

C.

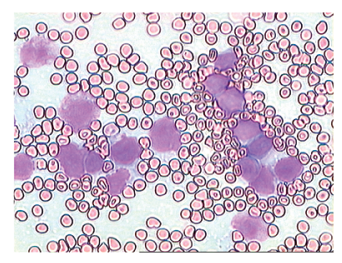

D.

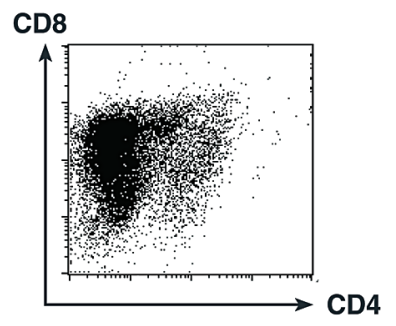

Figure 9. The invertor chromosomal translocation mimic. The invertor knockin translocation mimic involves introducing a cassette into the intron of a relevant gene. The invertor cassette comprises a short intronic sequence with an acceptor splice site, a segment of cDNA sequence for fusion with the targeted gene, and a poly(A) site. It is initially knocked-in in the opposite orientation for transcription (inactive form; $A$ ) and is flanked by lox $P$ sites to allow Cre-mediated inversion in Cre-expressing mice. After inversion, the acceptor site can be spliced to the donor splice site of the adjacent exon of the targeted gene ( $A$, active form). The $E W S$-ERG fusion found in human sarcoma was used to test this method. Ews-ERG invertor mice $(B)$ were bred with Ragl-Cre mice. Ews-ERG; Rag1Cre mice begin to show hematopoietic neoplasia after about 100 days $(B)$, developing T-cell tumors characterized by circulating lymphocytes ( $C$ shows a blood smear), mostly mature T cells ( $D$ shows an example of flow cytometry using CD8 and CD4 markers).

ons from the target gene and the cDNA from the invertor cassette.

As a model system to test the invertor method, we constructed a chromosomal translocation mimic of the EWS$E R G$ fusion. EWS is a translocation gene found in many human sarcomas and some leukemias, where it is fused to many different partners including ERG (Rabbitts 1994). A homologous gene FUS is also fused with $E R G$ in human acute myeloid leukemias. The invertor model was used to assess whether the Ews-Erg fusion could cause neoplasia in mice (Codrington et al. 2005; Forster et al. 2005). The invertor knockin cassette is depicted in Figure 9B. Cohorts of mice were established with the Ews-ERG or Ews-ERG plus a Rag1-Cre knockin allele (Fig. 9B, -Rag-Cre and + Rag-Cre, respectively) (McCormack et al. 2003). Leukemias developed in the Ews-ERG; Rag1Cre invertor mice, starting around 110 days; by 500 days, $>90 \%$ of these mice had succumbed to leukemia (Fig. 9B). The neoplasias were diagnosed as large-cell anaplastic T-cell lymphoma by a number of criteria, including presence of high numbers of circulating lymphocytes (Fig. 9C) and expression of markers, such as CD8, characteristic of mature T cells (Fig. 9D).

These data both validate the invertor model as a Credependent chromosomal translocation mimic (Forster et al. 2005) and show that the Ews-ERG fusion protein can be oncogenic in committed cells (Codrington et al. 2005).

\section{CONCLUSIONS}

We have developed three methods to make chromosomal translocation mimics in mice via homologous recombination in ES cells; namely, knockin, translocator, and invertor mice. Our mouse models with these approaches are summarized in Table 1. We have applied the gene knockin approach to one model (viz. Mll-AF9). Conditional forms using the invertor approach as a universal translocation mimic have induced lymphoid tumors with this method using Ews-ERG, Bcr-ABL, and Mll-AF4 fusions. The most faithful recapitulation of the reciprocal translocations found in human hematopoietic and mesenchymal neoplasias occurs in the translocator model where loxP sites on two (non-)homologous chromosomes serve as recombination signals for interchromosomal translocations. We are currently developing second-generation translocator models designed to accelerate the process of creating the translocator lines of mice and introducing elements in the design that will facilitate creating transcriptional options on both reciprocal chromosomes.

An interesting point of similarity exists between our results with mouse $M l l$ translocators and the $M L L$ fusion genes in man. There are a large number of different translocations to $M L L$ on human chromosome 11, and indeed, almost every chromosome arm has been implicated in $M L L$-associated leukemias. Thus, chromosome terri- 
Table 1. Gene Targeting Approaches to Chromosomal Translocations Mimics Result in Hematopoietic Tumors in Mice

\begin{tabular}{|c|c|c|c|}
\hline $\begin{array}{l}\text { Targeting } \\
\text { strategy }\end{array}$ & $\begin{array}{l}\text { Gene } \\
\text { fusion }\end{array}$ & $\begin{array}{l}\text { Cre } \\
\text { expressor }\end{array}$ & $\begin{array}{l}\text { Neoplasia (Bethesda } \\
\text { classification) }\end{array}$ \\
\hline \multicolumn{4}{|l|}{ Knock-in } \\
\hline & Mll-AF9 & N.A. & acute myeloid leukemia \\
\hline \multicolumn{4}{|l|}{ Translocator } \\
\hline & Mll-Afg & Lmo2-Cre & $\begin{array}{l}\text { MPD-like acute myeloic } \\
\text { leukemia }\end{array}$ \\
\hline & Mll-Enl & Lmo2-Cre & $\begin{array}{l}\text { MPD-like acute myeloic } \\
\text { leukemia }\end{array}$ \\
\hline & Mll-Enl & Lck-Cre & $\begin{array}{l}\text { small T-cell lymphoma } \\
\text { or myeloid leukemia } \\
\text { without maturation }\end{array}$ \\
\hline \multicolumn{4}{|l|}{ Invertor } \\
\hline & \multicolumn{2}{|c|}{ Ews-ERG Ragl-Cre } & $\begin{array}{l}\text { large-cell anaplastic } \\
\text { T-cell lymphoma }\end{array}$ \\
\hline & Mll-AF4 & Ragl-Cre & $\begin{array}{l}\text { diffuse large B-cell } \\
\text { lymphoma }\end{array}$ \\
\hline & Mll-AF4 & Cd19-Cre & $\begin{array}{l}\text { diffuse large B-cell } \\
\text { lymphoma }\end{array}$ \\
\hline & Mll-AF4 & Lck-Cre & $\begin{array}{l}\text { diffuse large B-cell } \\
\text { lymphoma }\end{array}$ \\
\hline & $B c r-A b l$ & $\mathrm{Mx}$-Cre & B-cell acute leukemia \\
\hline
\end{tabular}

N.A. indicates not applicable.

tory does not seem to impose much restriction in interchromosomal events in the precursor cell of human leukemias. Our data in the mouse models suggest similar flexibility. We find that chromosomal translocations occur with comparable efficiency between $M l l$ and Enl or Af9 genes, all located on separate chromosomes. In human cancer, selection processes presumably engender tumor appearance from rare cells in which a chance, but low probability, chromosomal translocation occurs. This cannot be so in the mouse translocators, since we can observe chromosomal translocations between $M l l$ and Enl and between $M l l$ and $A f 9$ within days after birth. In all, this suggests fluidity with which chromosomes are able to interact.

Mouse models of human cancer are relevant for the elucidation of factors influencing features of disease but are also important for developing rational therapeutic strategies and testing macromolecular drugs. With the advent of efficient recombination systems in mice, we have developed compound genetic systems to mimic the formation and effects of chromosomal translocations in human cancer. We have described three methods for this objective and hope that the next few years will see the use of these models as preclinical settings for drug development and validation.

\section{ACKNOWLEDGMENTS}

We thank the Medical Research Council for funding the work described in this paper. R.C. was a Leukaemia Research Fund Gordon Pillar Student, N.C. was a Croucher Foundation student, and F.C. is a C. Milstein Memorial student of the Darwin Trust, Edinburgh. N.L. was funded by the Kay Kendall Leukaemia Fund and by the Leukaemia Research Fund, M.M. by the German Research Foundation, C.H.N. by the Kay Kendall Leukaemia Fund and by the Lady Tata Memorial Fund,
S.R. by the Ministerio de Educación y Ciencia, and T.T. by the National Foundation for Cancer Research. We thank Ms. A. Lenton for the illustration work.

\section{REFERENCES}

Boveri T. 1914. Zur Frage der Entstehung Maligner Tumoren. Gustav Fischer, Germany.

Cleary M.L. 1991. Oncogenic conversion of transcription factors by chromosomal translocations. Cell 66: 619 .

Codrington R., Pannell R., Forster A., Drynan L.F., Daser A., Lobato M.N., Metzler M., and Rabbitts T.H. 2005. The EwsERG fusion protein can initiate neoplasia from lineage committed haematopoietic cells. Publ. Lib. Sci. Biol. 3: 242.

Collins E.C., Pannell R., Simpson E.M., Forster A., and Rabbitts T.H. 2000. Inter-chromosomal recombination of $M l l$ and $A f 9$ genes mediated by cre-loxP in mouse development. EMBO Rep. 1: 127.

Corral J., Lavenir I., Impey H., Warren A.J., Forster A., Larson T.A., Bell S., McKenzie A.N.J., King G., and Rabbitts T.H. 1996. An Mll-Af9 fusion gene made by homologous recombination causes acute leukemia in chimeric mice: A method to create fusion oncogenes. Cell 85: 853.

Dobson C.L., Warren A.J., Pannell R., Forster A., Lavenir I., Corral J., Smith A.J.H., and Rabbitts T.H. 1999. The Mll-AF9 gene fusion in mice controls myeloproliferation and specifies acute myeloid leukaemogenesis. EMBO J. 18: 3564.

Forster A., Pannell R., Drynan L.F., McCormack M., Collins E.C., Daser A., and Rabbitts T.H. 2003. Engineering de novo reciprocal chromosomal translocations associated with $\mathrm{Mll}$ to replicate primary events of human cancer. Cancer Cell 3: 449 .

Forster A., Pannell R., Drynan L.F., Codrington R., Daser A., Metzler M., Lobato M.N., and Rabbitts T.H. 2005. The invertor knock-in conditional chromosomal translocation mimic. Nat. Methods 2: 27.

Hanahan D. and Weinberg R.A. 2000. The hallmarks of cancer. Cell 100: 57.

Hoess R.H., Ziese M., and Sternberg N. 1982. P1 site-specific recombination: Nucleotide sequence of the recombining sites. Proc. Natl. Acad. Sci. 79: 3398.

Kogan S.C., Ward J.M., Anver M.R., Berman J.J., Brayton C., Cardiff R.D., Carter J.S., de Coronado S., Downing J.R., Fredrickson T.N., Haines D.C., Harris A.W., Harris N.L., Hiai H., Jaffe E.S., MacLennan I.C., Pandolfi P.P., Pattengale P.K., Perkins A.S., Simpson R.M., Tuttle M.S., Wong J.F., and Morse H.C. 2002. Bethesda proposals for classification of nonlymphoid hematopoietic neoplasms in mice. Blood 100: 238.

McCormack M.P., Forster A., Drynan L.F., Pannell R., and Rabbitts T.H. 2003. The LMO2 T-cell oncogene is activated via chromosomal translocations or retroviral insertion during gene therapy but has no mandatory role in normal T-cell development. Mol. Cell. Biol. 23: 9003.

Rabbitts T.H. 1991. Translocations, master genes, and differences between the origins of acute and chronic leukemias. Cell 67: 641.

. 1994. Chromosomal translocations in human cancer. $\mathrm{Na}$ ture 372: 143.

Rabbitts T.H. and Stocks M.R. 2003. Chromosomal translocation products engender novel intracellular therapeutic technologies. Nat. Med. 9: 383 .

Ramirez-Solis R., Liu P., and Bradley A. 1995. Chromosome engineering in mice. Nature 378: 720.

Smith A.J.H., De Sousa M.A., Kwabi-Addo B., Heppell-Parton A., Impey H., and Rabbitts P.H. 1995. A site-directed chromosomal translocation induced in embryonic stem cells by Cre-loxP recombination. Nat. Genet. 9: 376.

Tanaka T. and Rabbitts T.H. 2003. Intrabodies based on intracellular capture frameworks that bind the RAS protein with high affinity and impair oncogenic transformation. EMBO J. 22: 1025 .

Tanaka T., Lobato M.N., and Rabbitts T.H. 2003. Single domain intracellular antibodies: A minimal fragment for direct in vivo selection of antigen-specific intrabodies. J. Mol. Biol. 331: 1109. 
Tse E., Lobato M.N., Forster A., Tanaka T., Chung G.T.Y., and Rabbitts T.H. 2002. Intracellular antibody capture technology: Application to selection of single chain Fv recognising the BCR-ABL oncogenic protein. J. Mol. Biol. 317: 85.

van Deursen J., Fornerod M., van Rees B., and Grosveld G.
1995. Cre-mediated site-specific translocation between nonhomologous mouse chromosomes. Proc. Natl. Acad. Sci. 92: 7376.

$\mathrm{Yu}$ Y. and Bradley A. 2001. Engineering chromosomal rearrangements in mice. Nat. Rev. Genet. 2: 780. 


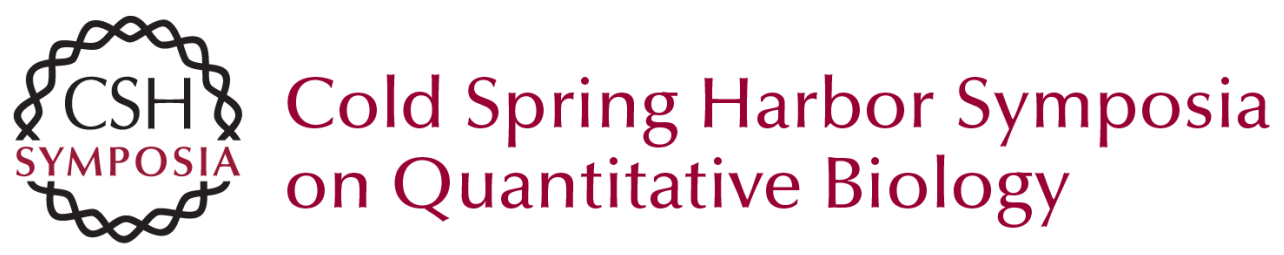

\title{
Chromosomal Translocation Engineering to Recapitulate Primary Events of Human Cancer
}

\author{
A. FORSTER, R. PANNELL, L. DRYNAN, et al.
}

Cold Spring Harb Symp Quant Biol 2005 70: 275-282

Access the most recent version at doi:10.1101/sqb.2005.70.008

References This article cites 21 articles, 6 of which can be accessed free at:

http://symposium.cshlp.org/content/70/275.full.html\#ref-list-1

License

Email Alerting Receive free email alerts when new articles cite this article - sign up in

Service the box at the top right corner of the article or click here. 